\title{
Microfluidics-based Low-Cost Medical Diagnostic Devices: Some Recent Developments
}

\author{
Shantimoy $\operatorname{Kar}^{1} \cdot$ Tapas Kumar Maiti ${ }^{1,2} \cdot$ Suman Chakraborty $^{1,3}$
}

Received: 4 April 2016/Accepted: 30 June 2016/Published online: 2 August 2016

(C) Indian National Academy of Engineering 2016

\begin{abstract}
Here we present a short review of recently developed low-cost microfluidic devices aimed towards medical diagnostic applications. This review specifically focuses on three inexpensive devices, namely lab-on-acompact-disc, paper-based, and thread-based devices. Here, we present a concise summary of all the recently developed protocols for simple bioassays to complex diagnostics. We also provide a new outlook on how the present practice of pathological diagnostics can be improved with the usage of such recent developments.
\end{abstract}

Keywords Microfluidics · Inexpensive · Diagnosis

\section{Introduction}

In accordance with World Health Organization (WHO)'s report (Observatory 2015), life expectancy of human beings has increased significantly throughout the globe. Thus, it is becoming a tough challenge for each nation to provide quality healthcare to its population. Involved clinical practices often follow tedious laboratory protocols like clinical chemistry, hematology, and immunology etc., which require the usage of expensive chemicals, sophisticated instruments, and trained personnel. In many

Suman Chakraborty

suman@mech.iitkgp.ernet.in

1 Advanced Technology Development Centre, Indian Institute of Technology Kharagpur, Kharagpur 721302, India

2 Department of Biotechnology, Indian Institute of Technology Kharagpur, Kharagpur 721302, India

3 Department of Mechanical Engineering, Indian Institute of Technology Kharagpur, Kharagpur 721302, India scenarios, the sample collected from the patient needs to be transferred to a specialized laboratory from a remote place. Even, the patient may need to be physically transferred to a sophisticated healthcare centre for diagnosis. Furthermore, the duration of analysis in traditional diagnostics may range from few hours to days. Thus there are urgent needs of fast, easy and affordable diagnostic devices and/or protocols. In this context, microfluidic technologies have found significant potential in terms of providing low-cost, rapid and yet efficient diagnostics (Pai et al. 2012; Gubala et al. 2012).

Typical microfluidic devices for medical diagnostics can be classified into two broad categories. One category involves the fabrication based on glass, silicon, PDMS or other polymer-based substrates, which often require expensive instruments and clean room facilities; thus may not suit for on-field applications.

Recent developments of lab-on-a-compact disc (CD) (Gorkin et al. 2010), paper-based (Gubala et al. 2012) and thread (specifically made of cotton)-based ( $\mathrm{Li}$ et al. 2010) microfluidic devices hold the potential to alter the scenario of point-of-care (POC) diagnostics (i.e. diagnostic activities on patient's site, and not in the laboratory). In this letter, we outline the recent developments on all three aforesaid devices for several fluidic functions and complex biological assays.

\section{CD-based Devices}

CD-based devices are explored for successful demonstration of several fluidic functions like mixing (Chakraborty et al. 2011; Ducrée et al. 2005), valving, separation (Zhang et al. 2008; Kuo and Li 2014; Kim et al. 2013a), bubble (Chakraborty and Chakraborty 2010)/droplet (Haeberle 
et al. 2007) generation. Operational details of such devices have been elaborately demonstrated by Madou et al. (2006) and its function as a potential bio-medical platform (Gorkin et al. 2010; Ducrée et al. 2007) has been well documented in literature. CD-based devices may be fabricated following different approaches. For example, one may adopt a layer-stacking method (Madou et al. 2006; Kar et al. 2015a), by which three PMMA sheets are attached with two pressure sensitive adhesives (PSA) layers and thus circumventing the usage of expensive instruments and intensive labor. In such devices, micro-channels are engraved within the adhesive layers. Fluid transportation through such devices is mainly guided by rotational actuation and thus diminishes the usage of expensive syringe pumps or other actuation mechanisms.

Complex biological assay like nucleic acid analysis (Kim et al. 2014), enzyme linked immunosorbent assay (ELISA) (Lai et al. 2004) have been explored on CD-based devices by judicious tailoring of rotational speeds. To improve the sensitivity of detection, Kim et al. demonstrated an electrochemical immunosensor (Kim et al. 2013b) which detects the bio-analytes at $\mathrm{pg} / \mathrm{mL}$ level. Efficient separation of blood plasma (Zhang et al. 2008; Kuo and Li 2014) from whole blood has been illustrated on CD-based platform, simply with the controlled interplay of channel geometry and as well as the rotational speeds. Centrifugally-actuated platforms have been delineated for high throughput screening and multiplexing (Park et al. 2012), and thus hold the potential of performing batch experiments on a single device.

Our research group initially focused on exploring several fluidic functions like capillary filing dynamics (Chakraborty et al. 2009), mixing behavior (Chakraborty et al. 2011), and generation of micro-bubble (Chakraborty and Chakraborty 2010), droplets to serpentine threads (Kar et al. 2015b) on CD-based platforms by exploiting the inter-playing physical forces. Currently, we are exploring this particular device for diagnostic applications. Our recent study reveals that on a rotating platform, the fluidic parameters are significantly altered with the change in the hematocrit value of blood (Kar et al. 2015a). In our case, we have characterize the blood dynamics in terms of two fluidic parameters namely burst frequency $\left(f_{b}\right)$ (i.e. the minimum rotational speed which is required to overcome the surface tension force or below which the fluid column will not burst into the channel from the inlet reservoir) and volumetric flow rate $(Q)$. In our experimentation, the used device has the dimension of length $(L)=2.9 \mathrm{~cm}$, height $(H)=100 \mu \mathrm{m}$, whereas width $(W)$ varies from 200 to $700 \mu \mathrm{m}$. For the characterization of blood dynamics, rotational speed of the device has been controlled in the range of 100-500 rpm. In our experimentation, alteration in the hematocrit value of blood, in turn, alters the rheological behavior of blood to a significant extent. This affects the fluidic parameters (Fig. 1 shows that the burst frequency is increasing with the increase of hematocrit value of blood). In another recent study, we have illustrated the effect of rotational stress on RBC's morphology (Kar et al. 2015c) (i.e. at cellular level). To characterize the RBC's morphological alterations, we define a parameter, named 'deformability index (DI)' (i.e. the ratio of major and minor axes of the ellipse while the RBCs are mapped as an ellipse). Hemoglobin, being an internal constituent of the RBCs, has significant influence on dictating the RBC's morphology (see Fig. 2). When the hemoglobin content is less than the standard value, then the cytoplasmic viscosity is expected to be less, which is manifested from the lesser value of DI. From our investigation, we envision that such alteration of fluidic parameters, or measurement of deformability indices can be used as inexpensive alternative to the conventional diagnostic approach for diagnosing hematological disorders.

\section{Paper-based Devices}

With an aim of providing 'zero cost diagnostics' Whitesides and co-researchers have first illustrated the fabrication and functions of paper-based devices (Martinez et al. 2010). Paper devices are fabricated by creating hydrophobic barriers, which direct the transportation of fluids in desired direction. To fabricate hydrophobic barriers on paper substrates, several techniques [like usage of

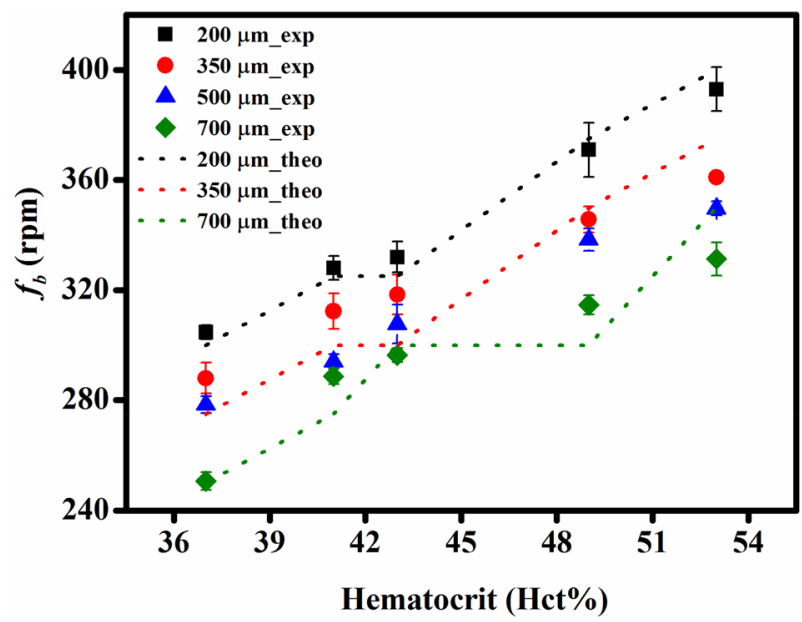

Fig. 1 Effect of hematocrit on burst frequency. The theoretical and experimental findings are merged in the same figure. Scattered data points represent the experimental findings whereas the dotted lines represent the theoretical results. The error bars indicate standard deviation (SD) of the results from repeated set of experiments (reproduced from Ref. Kar et al. (2015) with due permission from Royal Society of Chemistry, the article can be found http://pubs.rsc. org/en/content/articlehtml/2015/an/c4an02020k) 


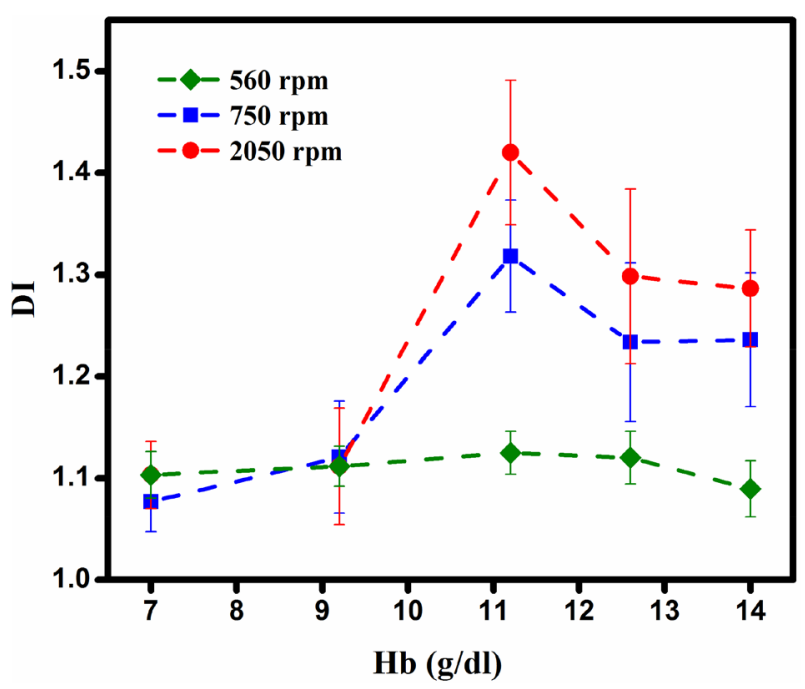

Fig. 2 Deformation characteristics as a function of hemoglobin content of the blood samples for different rotational speeds. The error bars represent the standard deviations (SD) of the results obtained from four repeated sets of experiments (reproduced from Ref. Kar et al. (2015) with due permission from Royal Society of Chemistry, the article can be found http://pubs.rsc.org/en/content/articlehtml/ 2015/lc/c5lc00968e)

epoxy-resin material, inkjet-printing, wax-printing (Carrilho et al. 2009), laser-cutting etc.] have been employed. Recently, we have developed a simple fabrication methodology (Dey et al. 2015), simply by using an office printer and a hot-plate. Through that particular modality of fabrication, necessary design can be fabricated by taking printing on both sides of the paper, followed by a heating at $200{ }^{\circ} \mathrm{C}$. This leads to the melting of toner particles deposited over the paper surfaces. These particles eventually impregnate within the paper, and thus form the hydrophobic barrier. Accordingly, fluid flow is directed along a desired path, primarily by capillary action.

Paper matrix is composed of enormous number of cellulose fibers. The fluid imbibes through such porous networks due to the capillary force. Such tortuous networks of fibres provide rudimentary control over the fluid flow. To achieve better reproducibility and excellent control over the fluid transportation through paper matrix, researchers have introduced several external actuation mechanism like surface acoustic waves (SAW) (Rezk et al. 2012), electric fields (Dey et al. 2015; Mandal et al. 2012), and centrifugal force (Hwang et al. 2011) etc. Considering the inexpensiveness and ease of fabrication of the paper-based devices, our group has shown the use of graphite electrodes simply by rubbing a tip of a pencil on paper, in an effort to have rapid capillary transport with the aid of electric field in a low cost paradigm. It is shown that the fluid transportation rate can be significantly enhanced with the introduction of external electrical actuation on 'paper-and-pencil' devices
(Mandal et al. 2012). Thereafter, we have demonstrated controlled mixing of two analytes on a zigzag 'paper-andpencil' device with the combined interplay of capillary and electrokinetic effect (Dey et al. 2015).

Paper-based devices have been widely used for several investigations ranging from diagnosis to quality control of foods (Jokerst et al. 2012) and drinking water. Colorimetric approach (Dungchai et al. 2010), fluorescence-based approach, electrochemical (Rattanarat et al. 2012; Dungchai et al. 2009) and electro-chemiluminescence (Zhang et al. 2013) approach have been employed to detect level of analytes. Amongst all the aforesaid approaches, colorimetric approach received wide acceptability due to its simplicity and involvement of minimal instrumentation. There are reports where people have explored the detection of bromide ions from natural water (Loh et al. 2015) and other heavy toxic metal ions from the drinking water (Zhang et al. 2013) on simple paper-based platforms at $\mathrm{ppm} / \mathrm{ppb}$ level. Another notable feature of this specific device is that it can easily be designed for diagnosing multiple analytes simultaneously on a single platform. It has been used for ELISA (Cheng et al. 2010), DNA hybridization, protein assay, anti-microbial activities (Kalsi et al. 2015), 'H-filter' devices for separation (Osborn et al. 2010) and lateral flow strip assays etc.

To avoid complexities while designing the devices, spot diagnosis is always favoured with colorimetric approach. One of the major issues while working with the colorimetric approach is that the intense red colour of the blood interferes in colorimetric detection. Thus, it is essential to separate the RBCs to diagnose any bio-analyte from plasma itself. Researchers have demonstrated efficient separation of blood plasma using a particular membrane (Songjaroen et al. 2012) (which specifically allow RBCs to adhere on its surface) and agglutination reagent (Yang et al. 2012). Very recently, we have demonstrated a simple paper-based 'H-channel' device for separating plasma (shown in Fig. 3) where we have reported an inexpensive modality for efficient separation of blood plasma using the capillarity-driven transportation of blood (Kar et al. 2015d). Using our approach, we have achieved 75\% separation of plasma. A recent work delineates plasma separation using a 'salt-functionalized' paper (Nilghaz and Shen 2015) where the adhesion properties of red blood cells (RBCs) were augmented due usage of salt, and thus plasma gets separated from blood.

The colorimetric approach is globally used for qualitative and quantitative detection. To enhance the sensitivity of the detection, nanoparticles impregnated paper ( $\mathrm{Fu}$ et al. 2011; Fu et al. 2010) have been used. Later on, researchers have attempted to employ 'Bioplasmonic paper (i.e. paper impregnated with nanoparticles having plasmon resonance)' for kidney biomarker detection (Tian et al. 2012). 


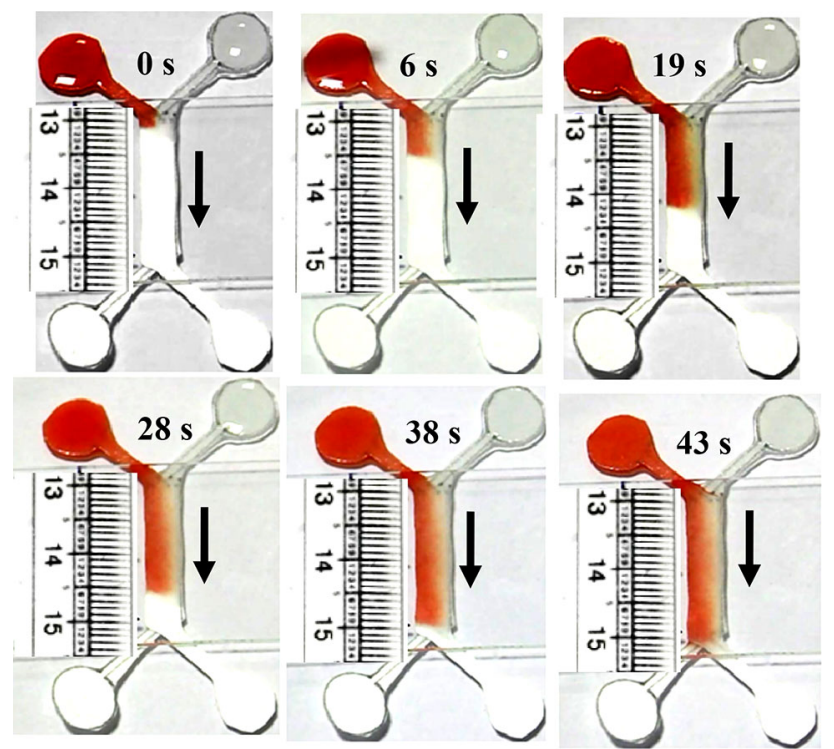

Fig. 3 Real time image sequences showing how the blood stream diffuses into the buffer stream and accordingly the blood strain covers the entire width of the paper channel (reproduced from Ref. Kar et al. (2015) with due permission from Royal Society of Chemistry, the article can be found http://pubs.rsc.org/en/content/articlehtml/2015/ an/c5an00849b)

With this approach, they have diagnosed Hepatitis B virus DNA using electrochemical paper sensor ( $\mathrm{Li}$ et al. 2015). Yildiz et al. have demonstrated a simple paper-based biosensing platform for colorimetric detection of lung cancer associated miRNA (Yildiz et al. 2013).

Three dimensional (3D) paper devices have been fabricated by using paper and tape (Martinez et al. 2008) and also following an origami-based protocol (Liu and Crooks 2011; Scida et al. 2013), and same has been utilized for several applications (Scida et al. 2013; Liu et al. 2012; Ge et al. 2012). There are other reports which discuss about hollow paper channels (Renault et al. 2013), which have also been used for bio-analyte detection.

It is important to note here that the applications of such paper-based devices are not only limited in the field of diagnostics, but have also been extended to address diverse applications like titration (Myers et al. 2015; Karita and Kaneta 2014), power generation (Arun et al. 2014; Veerubhotla et al. 2015) (fuel-cells), and the development of flexible electronics (Kurra et al. 2013; Kurra and Kulkarni 2013) etc.

\section{Thread-based Devices}

In this section, we are going to briefly discuss about threadbased (made of cotton) inexpensive platform. Versatility of the thread-based devices in the context of developing diagnostic devices has been established by Li et al. (2010). Thereafter in 2011, Ballerini et al. have demonstrated the usage of thread-based devices for mixing, and acid-base titration (Ballerini et al. 2011). There are reports which show several fluidic functions (Taylor et al. 2012) by using yarns and knots (Safavieh et al. 2011) with the thread. Recently, complex functions like immune-chromatographic assay on threads (Zhou et al. 2012) has been illustrated by Zhou et al. Human ferittin has been successfully diagnosed by performing immunoassay on cotton-threads (Mao et al. 2015).

\section{Conclusions}

In this brief review, we have summarized some recent developments on low-cost microfluidic devices for pointof-care medical diagnostics. All the three devices discussed here, namely lab-on-a-CD, paper based devices, and thread based devices are easy to manufacture, portable, multiplexable (i.e. multiple analytes can be simultaneously diagnosed on a single platform), easily disposable, affordable for the mass population, easily operated from resource limited places, and are therefore appropriate for on-field trials. These types of affordable diagnostic platforms can be very useful for on-spot diagnostics and as well as for continuous monitoring for a particular disease condition. Though the several complex bioassays have been demonstrated on simple platforms but such devices are yet to be popularized in mass scale, especially in developing nations. We envision that these devices hold the potential of replacing the traditional laboratory-centric, expensive, and time intensive paradigm of medical diagnostics. One primary bottleneck towards that is an appropriate upscaling of the these devices from the laboratory scale to the commercial scale, as well as establishing the reliabilities of the read-outs from such devices to all the stake holders under concern. Further, one may integrate the low cost hardware of these devices with Smartphone technology (mobile apps) for integrating with the telemedicine facilities available at resource-limited locations. Efforts towards that direction are currently under way.

Acknowledgments SK greatly acknowledges to Council of Scientific and Industrial Research (CSIR), India for his research fellowship.

\section{References}

Arun RK et al (2014) A paper based self-pumping and self-breathing fuel cell using pencil stroked graphite electrodes. Lab Chip 14(10):1661-1664

Ballerini DR, Li X, Shen W (2011) Flow control concepts for threadbased microfluidic devices. Biomicrofluidics 5(1):1-13 
Carrilho E, Martinez AW, Whitesides GM (2009) Understanding wax printing: a simple micropatterning process for paper-based microfluidics. Anal Chem 81:7091-7095

Chakraborty D, Chakraborty S (2010) Controlled microbubble generation on a compact disk. Appl Phys Lett 97(23):234103

Chakraborty D et al (2009) Capillary filling in centrifugally actuated microfluidic devices with dynamically evolving contact line motion. J Appl Phys 105(8):084904

Chakraborty D, Madou M, Chakraborty S (2011) Anomalous mixing behaviour in rotationally actuated microfluidic devices. Lab Chip 11(17):2823-2826

Cheng C-M et al (2010) Paper-based ELISA. Angewandte Chemie (Int Ed Engl) 122(28):4881-4884

Dey R et al (2015) Ultra-low-cost "paper-and-pencil" device for electrically controlled micromixing of analytes. Microfluid Nanofluid 19(2):375-383

Ducrée J et al (2005) Patterning of flow and mixing in rotating radial microchannels. Microfluid Nanofluid 2(2):97-105

Ducrée J et al (2007) The centrifugal microfluidic Bio-Disk platform. J Micromech Microeng 17(7):103-115

Dungchai W, Chailapakul O, Henry CS (2009) Electrochemical detection for paper-based microfluidics. Anal Chem 81(6): $5821-5826$

Dungchai W, Chailapakul O, Henry CS (2010) Use of multiple colorimetric indicators for paper-based microfluidic devices. Analytica Chimica Acta 674(2):227-233

Fu E et al (2010) Chemical signal amplification in two-dimensional paper networks. Sens Actuators B Chem 149(1):325-328

Fu E et al (2011) Enhanced sensitivity of lateral flow tests using a twodimensional paper network format. Anal Chem 83:7941-7946

Ge L et al (2012) Three-dimensional paper-based electrochemiluminescence immunodevice for multiplexed measurement of biomarkers and point-of-care testing. Biomaterials 33(4):10241031

Gorkin R et al (2010) Centrifugal microfluidics for biomedical applications. Lab Chip 10(14):1758-1773

Gubala V et al (2012) Point of care diagnostics: status and future. Anal Chem 84(2):487-515

Haeberle S, Zengerle R, Ducrée J (2007) Centrifugal generation and manipulation of droplet emulsions. Microfluid Nanofluid 3(1):65-75

Hwang H et al (2011) Paper on a disc: balancing the capillary-driven flow with a centrifugal force. Lab Chip 11(20):3404-3406

Jokerst JC et al (2012) Development of a paper-based analytical device for colorimetric detection of select foodborne pathogens. Anal Chem 84:2900-2907

Kalsi S et al (2015) Rapid and sensitive detection of antibiotic resistance on a programmable digital microfluidic platform. Lab Chip 15(14):3065-3075

Kar S, Dash M et al (2015a) Effect of hematocrit on blood dynamics on a compact disc platform. Analyst 140(5):1432-1437

Kar S, Joshi S et al (2015b) Generation of droplets to serpentine threads on a rotating compact-disk platform. Appl Phys Lett 107:244101

Kar S, Ghosh U et al (2015c) Haemoglobin content modulated deformation dynamics of red blood cells on a compact disc. Lab Chip 15(24):4571-4577

Kar S, Maiti TK, Chakraborty S (2015d) Capillarity-driven blood plasma separation on paper-based devices. Analyst 140:6473-6476

Karita S, Kaneta T (2014) Acid-base titrations using microfluidic paper-based analytical devices. Anal Chem 86:12108-12114

Kim TH et al (2013a) Geometry effects on blood separation rate on a rotating disc. Sens Actuators B Chem 178:648-655

Kim T-H et al (2013b) Flow-enhanced electrochemical immunosensors on centrifugal microfluidic platforms. Lab Chip 13(18): $3747-3754$
Kim T-H et al (2014) Fully integrated lab-on-a-disc for nucleic acid analysis of food-borne pathogens. Anal Chem 86:3841-3848

Kuo JN, Li BS (2014) Lab-on-CD microfluidic platform for rapid separation and mixing of plasma from whole blood. Biomed Microdev 16(4):549-558

Kurra N, Kulkarni GU (2013) Pencil-on-paper: electronic devices. Lab Chip 13(15):2866-2873

Kurra N, Dutta D, Kulkarni GU (2013) Field effect transistors and RC filters from pencil-trace on paper. Phys Chem Chem Phys 15(21):8367-8372

Lai S et al (2004) Design of a compact disk-like microfluidic platform for enzyme-lined immunosorbent assay. Anal Chem 76(7):1832-1837

Li X, Tian J, Shen W (2010) Thread as a versatile material for lowcost microfluidic diagnostics. ACS Appl Mater Interfaces 2(1): $1-6$

Li X, Scida K, Crooks RM (2015) Detection of hepatitis B virus DNA with a paper electrochemical sensor. Anal Chem 87(17): 9009-9015

Liu H, Crooks RM (2011) Three-dimensional paper microfluidic devices assembled using the principles of origami. J Am Chem Soc 133:17564-17566

Liu $\mathrm{H}$ et al (2012) Aptamer-based origami paper analytical device for electrochemical detection of adenosine. Angewandte Chemie Int Ed 51(28):6925-6928

Loh LJ et al (2015) Detection of water contamination from hydraulic fracturing wastewater: a $\mu \mathrm{PAD}$ for bromide analysis in natural waters. Analyst 140(16):5501-5507

Madou M et al (2006) Lab on a CD. Annu Rev Biomed Eng 8:601-628

Mandal P, Dey R, Chakraborty S (2012) Electrokinetics with "paperand-pencil" devices. Lab Chip 12(20):4026-4028

Mao X et al (2015) Novel gold nanoparticle trimer reporter probe combined with dry-reagent cotton thread immunoassay device for rapid human ferritin test. Analytica Chimica Acta 889:172-178

Martinez AW, Phillips ST, Whitesides GM (2008) Three-dimensional microfluidic devices fabricated in layered paper and tape. Proc Natl Acad Sci USA 105(50):19606-19611

Martinez AW et al (2010) Diagnostics for the developing world: microfluidic paper-based analytical devices. Anal Chem 82(1):3-10

Myers NM, Kernisan EN, Lieberman M (2015) Lab on paper: iodometric titration on a printed card. Anal Chem 87: 3764-3770

Nilghaz A, Shen W (2015) Low-cost blood plasma separation method using salt functionalized paper. RSC Adv 5(66):53172-53179

Observatory, G.H., 2015. Global Health Observatory., http://www. who.int/gho/publications/world_health_statistics/2016/EN_WHS 2016_Chapter3.pdf?ua=1. Accessed 16 July 2016

Osborn JL et al (2010) Microfluidics without pumps: reinventing the $\mathrm{T}$-sensor and H-filter in paper networks. Lab Chip 10(20): 2659-2665

Pai NP et al (2012) Point-of-care testing for infectious diseases: diversity, complexity, and barriers in low- and middle-income countries. PLoS Med 9(9):e1001306

Park J et al (2012) Lab-on-a-disc for fully integrated multiplex immunoassays. Anal Chem 84:2133-2140

Rattanarat P et al (2012) Sodium dodecyl sulfate-modified electrochemical paper-based analytical device for determination of dopamine levels in biological samples. Analytica Chimica Acta 744:1-7

Renault C et al (2013) Hollow-channel paper analytical devices. Anal Chem 85:7976-7979

Rezk AR et al (2012) Uniform mixing in paper-based microfluidic systems using surface acoustic waves. Lab Chip 12(4):773-779 
Safavieh R, Zhou GZ, Juncker D (2011) Microfluidics made of yarns and knots: from fundamental properties to simple networks and operations. Lab Chip 11(15):2618-2624

Scida K et al (2013) DNA detection using origami paper analytical devices. Anal Chem 85:9713-9720

Songjaroen $\mathrm{T}$ et al (2012) Blood separation on microfluidic paperbased analytical devices. Lab Chip 12(18):3392

Taylor P et al (2012) Textile-based microfluidics: modulated wetting, mixing, sorting, and energy harvesting. J Text Inst 103(December):37-41

Tian L et al (2012) Bioplasmonic paper as a platform for detection of kidney cancer biomarkers. Anal Chem 84:9928-9934

Veerubhotla $R$ et al (2015) Instant power generation from an airbreathing paper and pencil based bacterial bio-fuel cell. Lab Chip 15:2580-2583
Yang X et al (2012) Integrated separation of blood plasma from whole blood for microfluidic paper-based analytical devices. Lab Chip 12(2):274

Yildiz UH, Alagappan P, Liedberg B (2013) Naked eye detection of lung cancer associated miRNA by paper based biosensing platform. Anal Chem 85(2):820-824

Zhang $\mathrm{J}$ et al (2008) A lab-on-CD prototype for high-speed blood separation. J Micromech Microeng 18(12):125025

Zhang $M$ et al (2013) Three-dimensional paper-based electrochemiluminescence device for simultaneous detection of $\mathrm{Pb}^{2+}$ and $\mathrm{Hg}^{2+}$ based on potential-control technique. Biosens Bioelectron 41(2013):544-550

Zhou G, Mao X, Juncker D (2012) Immunochromatographic assay on thread. Anal Chem 84:7736-7743 